\title{
A NORMALIZED WEIGHTED ENTROPY MEASURE FOR SENSOR ALLOCATION WITHIN SIMULATIONS
}

\author{
Darryl Ahner \\ Dept. of Mathematical Sciences \\ United States Military Academy \\ West Point, NY, 10996, USA
}

\begin{abstract}
Information superiority is considered a critical capability for future joint forces. Sensor allocation and information processing are critical to achieving this information superiority but the value of information is difficult to assess. We develop a weighted entropy measure for sensor allocation within simulations by using the Dynamic Model of Situated Cognition as a framework in which to view the processing and flow of information in a complex technological-cognitive system. The entropy measure developed is normalized across each requirement and weighted according to the Commander's priorities within the phase of that operation. We develop a methodology for implementation for this normalized weighted entropy measure to allocate sensors within a combat simulation.
\end{abstract}

\section{INTRODUCTION}

Decision-makers struggle with the value of information in almost all forms. Therefore, it is not surprising that the way information is valued and used within combat simulations is also difficult to represent. In this paper we propose a methodology that relies on Commander's Critical Information Requirements (CCIR) that are defined in the planning stage and are linked to phases of combat operations. We particularly do not rely on the notion of the expected value of information which requires calculation but, instead, rely on a measure of uncertainty as it relates to mission priorities, namely, the weighted entropy measure which we describe.

Sensor allocation has increased in its importance as the use of sensors has increased with the proliferation of unmanned aerial vehicles, unmanned ground vehicles, unattended ground sensors and others. Methodologies such as the Assignment Scheduling Capability for Unmanned Aerial Vehicles (Ahner 2006) assigns sensors to demands but needs an external mechanism to assign the value received for a sensor-demand assignment. Sensor assets should be allocated based upon the extent that a sensor allocation reduces the uncertainty within this weighted entropy measure. Entropy, when used in the context of information, is often thought of in terms of Shannon's entropy measure which quantifies, in an expected value sense, the quality of long messages, usually in units such as bits. In this paper, we are not interested in the quality of the message but focus on the content of information as it applies to decision making. Nonetheless, we use a weighted entropy measure due to its excellent properties of measuring uncertainty of information.

Barr and Sherrill (1996) explore "information gain" in a military context with the addressed primary objective appearing to be "to study relationships between information gained about the enemy disposition and various measures of combat effectiveness (Barr and Sherrill 1996)." A Bayesian update is used to update the probability of an event given the probability that a sensor detects a target. This new probability is used to calculate the new entropy. The difference in the old and new entropy is what is referred to as "information gain." Our paper improves upon this concept by normalizing information gain across competing requirements for Reconnaissance Surveillance and Target Acquisition (RSTA) resources in support of CCIR.

The U.S. Army Combined Arms Combat Development Agency divided CCIR into eight new categories - intelligence, maneuver, battlefield geometry, fire support, air defense artillery, combat support, combat service support, and command guidance (Spinuzzi 2007). This paper focuses on information for intelligence that influences, as part of a larger system of systems, the other categories.

Several RAND studies address the value information and how they affect combat outcomes (Hammitt 1991, Gonzolez 2001). Research completed by the Naval Postgraduate School also tends to focus on outcomes (Ahner 2008). These RAND and NPS posterior measures aid in analysis but do not lend themselves to actively informing the act of gaining information to inform decisions. Unfortunately, attacking the value of information problem has proven difficult. As Washburn (2001) says, 
"There is a crisis for military OR, centered on the role of information on the battlefield. It is clear to military professionals that information is becoming increasingly important, but unfortunately the OR profession's ability to measure its contribution is still primitive." To address the role of information we look at it within the Dynamic Model of Situated Cognition (DMSC) (Shattuck and Miller 2006). The Dynamic Model of Situated Cognition (Figure 1) is a conceptual model that attempts to illustrate the relationship between technological systems and human perceptual and cognitive processes.

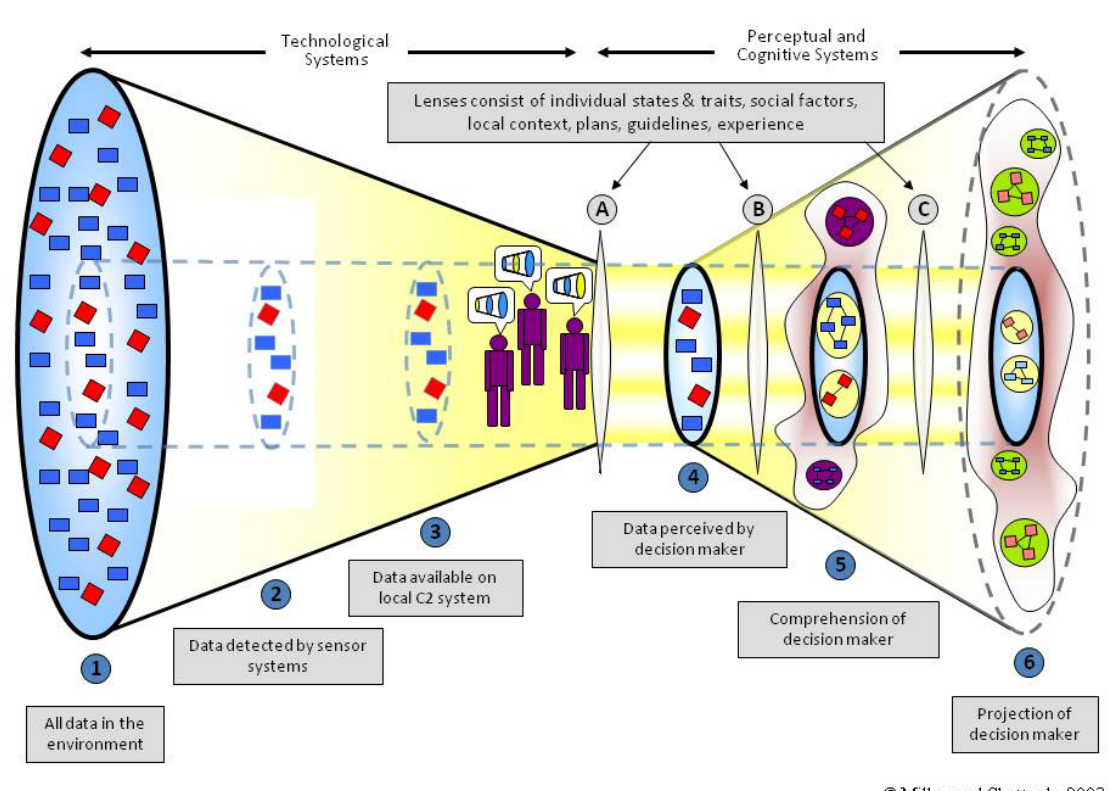

Figure 1: Dynamic Model of Situated Cognition

This paper presents an overview of the Dynamic Model of Situated Cognition and the elements of information and cognition that should be considered for development and inclusion within simulations, the decision problem to be addressed, Commander's Critical Information Requirements and the associated information vector, the methodology for measuring uncertainty using weighted normalized entropy, and some conclusions.

\section{THE DYNAMIC MODEL OF SITUATED COGNITION}

The Dynamic Model of Situated Cognition (Figure 1) is a conceptual model that attempts to illustrate the relationship between technological systems and human perceptual and cognitive processes. The left side of the model represents all data in the real world in oval 1, the data that is visible to deployed sensors in oval 2, and the information that is visible on local information systems in oval 3. We refer to the left side of the DMSC as the technological systems. The right side of the model represents the perceptual and cognitive systems. The right side represents perception in oval 4 , comprehension in oval 5 , and projection in oval 6 . Perception, comprehension, and projection are all needed to gain information superiority.

The lenses in the Dynamic Model of Situated Cognition combine elements from the left side of each lens with informational elements 'resident' in the decision maker and feed forward information or decisions to the next portion of the model. The decision maker's behavior is critical to understanding the data and information flow within the DMSC. The model is centered around the decision maker's information needs and information flow to the decision maker. Note that oval 2 is, in the best of cases, a subset of the first oval. Data elements that are the same in ovals 1 and 2 represent the accurate detection of items. We refer to this as technological accuracy. Not everything in Oval 1 is detected accurately for a variety of reasons. Sensors can either miss or misidentify data in Oval 1. Data may be missed for the following reasons:

- There may be an insufficient number of sensors to cover the environment.

- The technology may not be sensitive enough to detect certain classes of data.

- The technology may not have the specificity required to identify certain classes of data.

- Sensors may malfunction. 
Three dynamic lenses are depicted in Figure 1. The lenses in the Dynamic Model of Situated Cognition model combine elements from the left side of each lens with informational elements 'resident' in the decision maker and feed forward information or decisions to the next portion of the model. Situated cognition is not a state that is achieved but a dynamic, ongoing process (Clancy 1997). For example, the sensor coverage and communications network are dynamic. The sensor coverage is shifting constantly as sensors move or fly about the environment, resulting in changes to sensor coverage. These changes in coverage, will affect data available in Ovals 2 and 3 and ultimately influence the perception, comprehension, projection, and decision making. Developing a measure for effectively controlling sensor coverage for maximum information gain is the goal of the methodology that is developed.

Information does not just flow to one individual. The DMSC can also branch between oval 2 and oval 3 to provide differing $\mathrm{C} 2$ system representations to different users and illustrated for a commanders staff in Figure 2. These staff elements then feed into the Commander's perception, comprehension, and projection to provide a more robust understanding.

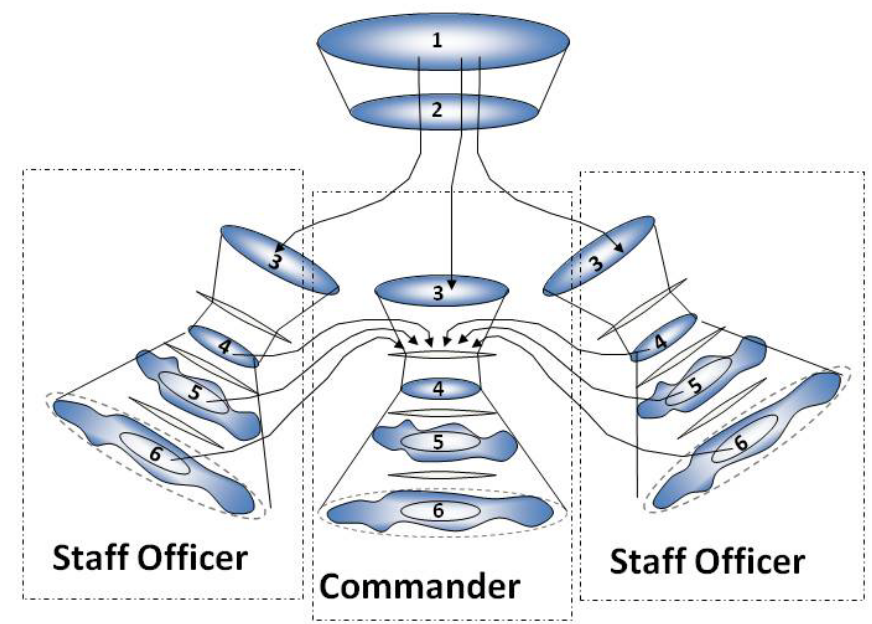

Figure 2: Dynamic Model Applied to Commander's Staff

Several analytical problems are embedded within the DMSC model that may need to be represented within simulations. As shown in Figure 3, these problems span differing portions of the DMSC model. Scarce sensor resources must be allocated and reallocated, required information must reach appropriate destinations, information fusion must occur to combine data into useable information, data association between sensor streams must occur to estimate the state of the system, and Commander's staff and Commander's must process this information through human cognition. A large portion of allocating resources to performing these functions and routing information involves correctly valuing information. Valuing information cover ovals 1 to ovals 5 and directly feeds into developing situational awareness. Solving each of these analytical problems lends itself to improving the likelihood for mission accomplishment. This paper looks at valuing information originating from sensors before they are allocated. Sensors can either be electronic device such as electro-optical, infrared, moving target indicator radar, signal intelligence etc. or human observation reports. These sensors have capabilities of detecting activity which will need to be quantified. 


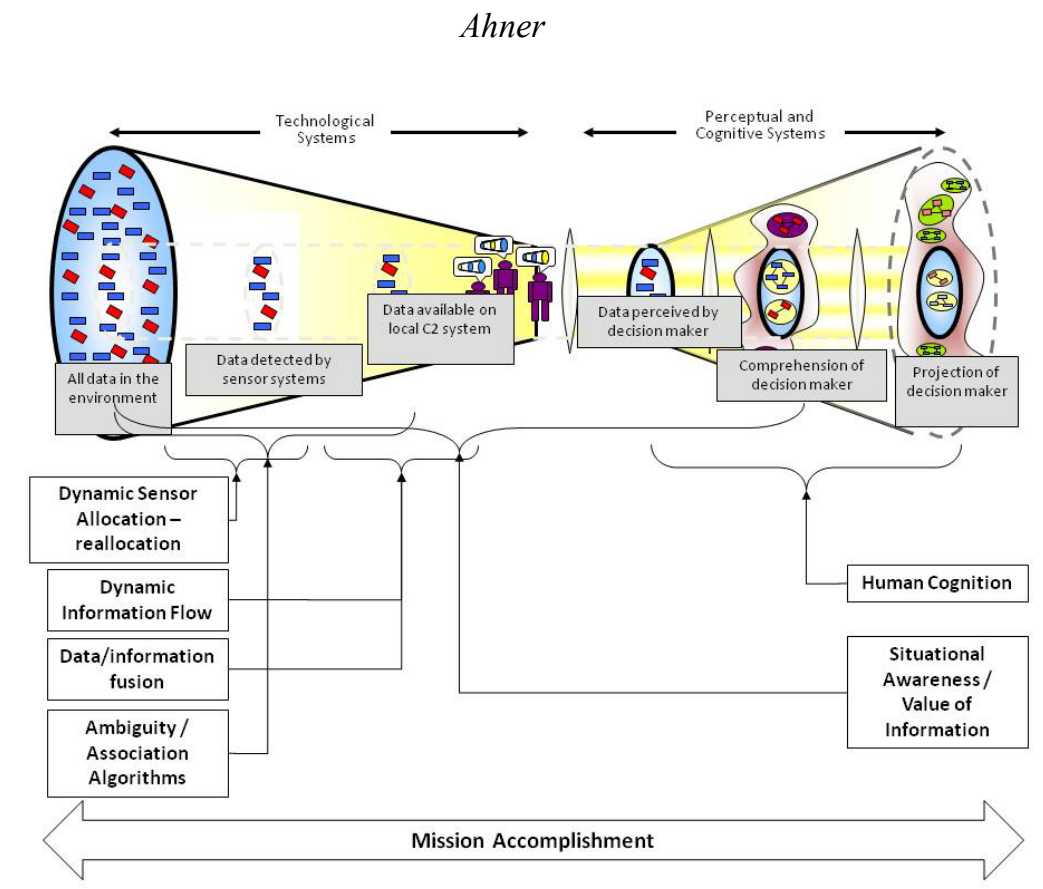

Figure 3: Problems of Interest within the Dynamic Model

\section{COMMANDER'S CRITICAL INFORMATION REQUIREMENTS}

Army Field Manual (FM) 2-0 Intelligence indicates the importance and use of information. "Commanders must have detailed information to command. Information is the medium that allows the commander's decisionmaking and execution cycle to function. Information gives direction to actions by the force, identifies the enemy's centers of gravity, provides COAs for force activity, and enables the force to accomplish its operational mission." In 1997, the U.S. Army introduced Commander's Critical Information Requirements (CCIR) as an essential component of Army Command and Control (C2) doctrine (FM 101-5). CCIR are "elements of information required by commanders that directly affect decisionmaking and dictate the successful execution of military operations (FM 6-0)." A RAND study "Understanding Commander's Information Needs" initially linked these elements to mission analysis (Kahan 1989).

Figure 4 shows the Intelligence Surveillance Reconnaissance (ISR) Task Development Process given in FM 2-0 within the dashed box with two exceptions. First, instead of feeding COA Analysis and Mission Analysis directly into Information Requirements, Situational Understanding is inserted. Second, the process now explicitly feeds into an iterative process within the FM. While the FM calls for the ISR Plan to be an annex in the Operations Order for units, this planning annex is simply a strawman for the updates that the FM doctrinally requires. Figure 4 simply takes the DMSC and the ISR Task Development Process and integrates them into a coherent iterative process that adapts as information becomes available and the state of the operation evolves.

The Task Development Process is extended into a dynamic process in Figure 4. The figure illustrates feedback through the Priority Intelligence Requirements (PIR) portion of the Commander's Critical Information Requirements (CCIR). CCIR are a list of information requirements identified by the commander as being critical in facilitating timely information management and the decision making process that affect successful mission accomplishment. The two key subcomponents are critical friendly force information and Priority Intelligence Requirements.

As the process begins, mission analysis occurs where higher headquarter's order is analyzed to determine what must be accomplished. Course of Action (COA) Analysis then occurs to put plans in place to accomplish the mission. These plans require information that must be obtained in spite of the influences of the environment and enemy actions. Intelligence Requirements are information elements required for planning and executing operations. While PIR are designated by the commander and are requirements associated with a decision that affects mission accomplishment. These intelligence requirements generate specific information requirements that are an identified gap in intelligence that may be satisfied only by collection action. These requirements generate task which are then satisfied by limited sensor assets in a developed ISR Plan.

Commander's intelligence requirements are tied directly to his decisions as CCIR (PIR and Friendly Force Information Requirements, FFIR). He uses them, within the mathematical cognitive model being developed, to improve situational under- 
standing by increasing information reliability and decreasing uncertainty through the more effective deployment / redeployment of organic sensors.

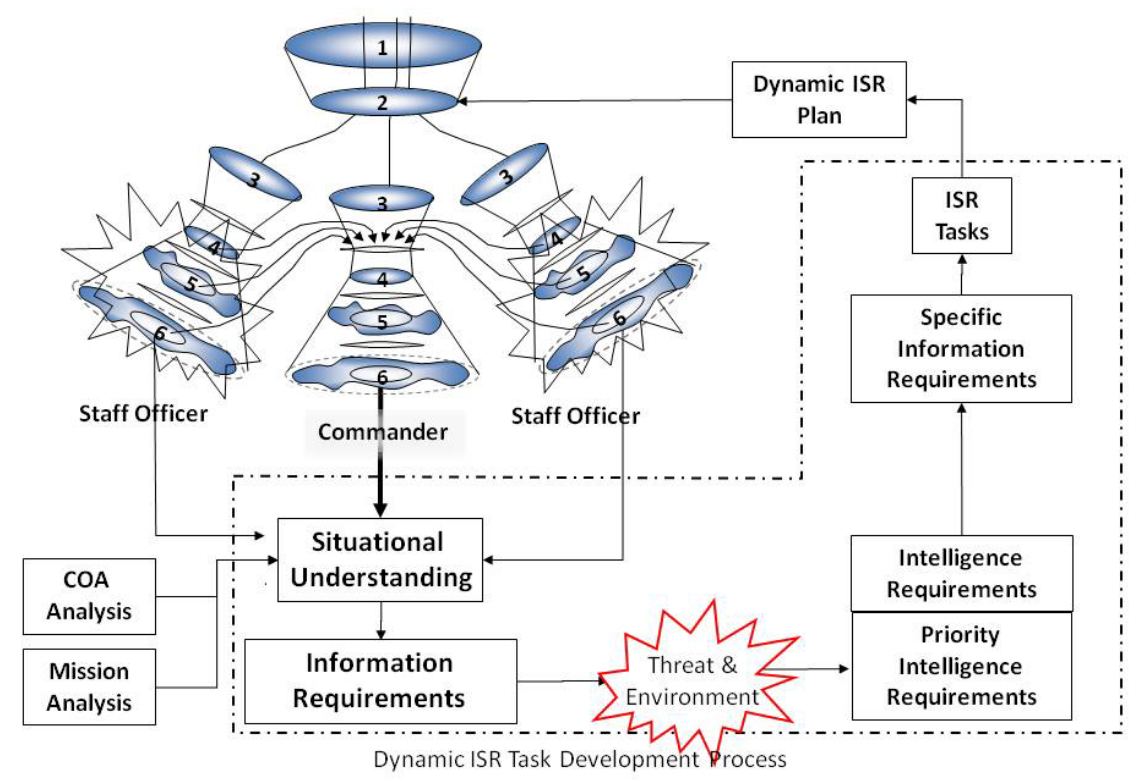

Figure 4: Dynamic ISR Task Development

In decision analysis, often influence diagrams are used that account for uncertainty, but the decision-maker has no control of the information that feeds that uncertainty (Howard 2005). The decision is based upon the decision-makers situational awareness which has many uncertainties. Among these uncertainties are missing data, reliability of the data, conflicting data, data timeliness, and ambiguous or noisy data (Endsley 2003). We now develop a the normalized weighted entropy measure for sensor allocation methodology that mitigates these uncertainties.

\section{METHODOLOGY}

The normalized weighted entropy measure for sensor allocation methodology, hereafter referred to as the Weighted Information Measure Methodology (WIMM), is structured around the Dynamic ISR Task Development Process in Figure 4. The process informs the requirements of an effective information measure used to support Commander's decisions.

We can look at the Commander's decision using decision theory. A decision problem, $\mathrm{D}$, is defined as $D=\langle A, X, \Omega, u, w, I, C(I)\rangle$.

Where the sets used are action space $(A)$, state space $(X)$, an outcome space $(\Omega)$ defined on a probability space $(W)$ such that the probability is defined on $X \times A$, the decision makers (DMs) utility function $(u)$, the initial wealth $(w)$, the available information about the random variables $(I)$, and an information cost function $(C(I))$.

The information structure available to the DM is $I=\{Y, p(x, y \mid x)\}$ where $x \in X$, all possible states and $y \in Y(x)$, the possible sensor messages.

Typically the probabilities within the information structure and the utility measure for a decision problem are assessed independently from one another. As a result, the expected utility of perfect information can be viewed as a real valued function of the probability outcomes where the optimal action changes with the discrete probability $p=p_{1}, p_{2}, \ldots p_{N}$. The decisionmaker does not make decisions according to the state space $x \in X$ rather according to information $(I)$ available about that state space. This information may or may not be accurate and complete.

Using this construct within our simulation we assume to have a decision engine that takes the information structure available to the DM and uses the state given by this information structure to determine the course of action chosen. The way in which this information structure is updated should directly relate to the decision being made. In order to accomplish this we use the actual method that is employed in the real world, Intelligence Preparation of the Battlefield. 


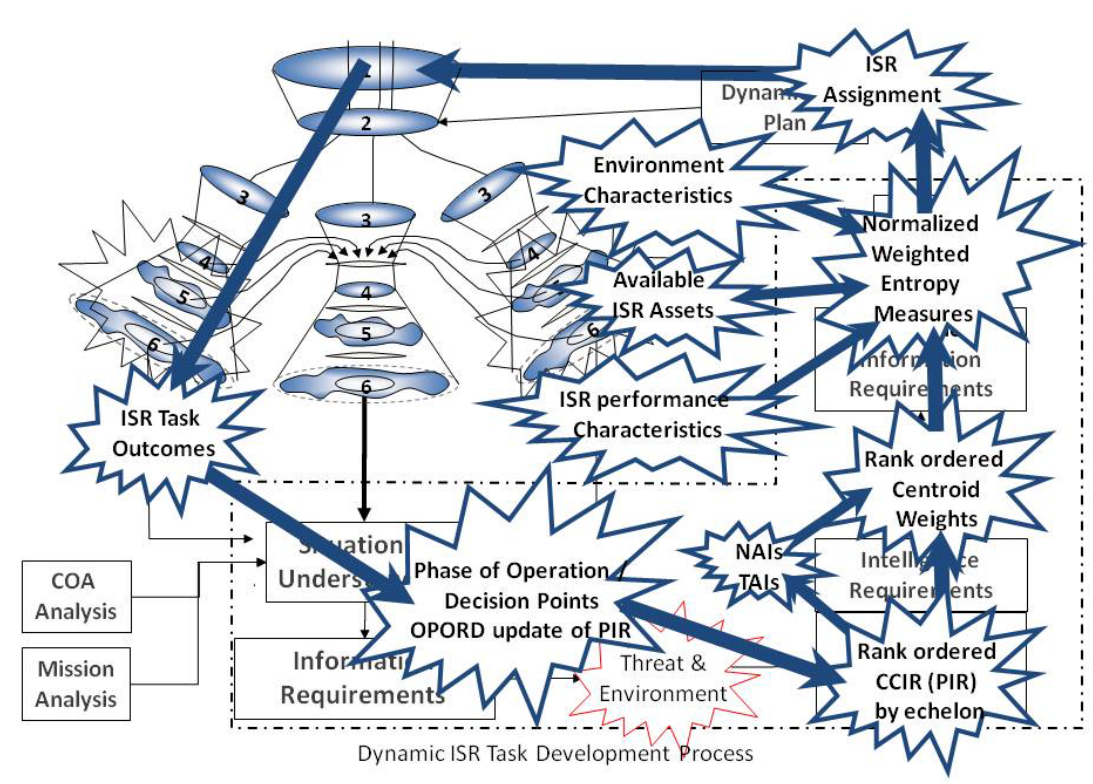

Figure 5: Weighted Information Measure Methodology to Support Commander's Decisions

The methodology begins using the products from the Intelligence Preparation of the Battlefield (IPB) as outlined in FM 2-0. IPB is a systematic process of analyzing and visualizing the threat and battlespace in a specific geographic area for a specific mission or in anticipation of a specific mission. IPB is conducted in simultaneously with course of action (COA) analysis and construction of the operations order (OPORD). Once an initial course of action is selected, the Commander identifies intelligence requirements associated with that COA and identities the most important as PIR. These PIR are part of the Commander Critical Information Requirements and must be prioritized in order for the WIMM to weigh the information gained by satisfying them. Given a prioritized list flowing from the phases of the operation, decision points, and OPORD updates as shown in Figure 5, Rank Order Centroid Weighting is used to assign weights.

\subsection{Rank Order Centroid Weighting}

Rank Order Centroid (ROC) weighting is a technique proposed for use in decision analysis to weight criteria (Edwards and Barron 1994, Barron and Barrett 1996). Barron \& Barrett demonstrate that ROC weighting is straightforward and efficacious. The mathematical objective is to derive relative values for the local groupings of criteria such that the sum of the values equal one and that the individual values are indicative of the priorities associated with the criteria under consideration (i.e., $\Sigma v_{i}=1$ and $v_{1}>v_{2}>\ldots>v_{n}$ ). The ROC method has two favorable characteristics: ease of elicitation from decision makers and minimal loss of information as ranking judgments are transformed into normalized weights summing to one. While the Commander may not have prioritized all their PIR, if the Commander or their staff prioritizes their Named Area of Interest (NAI) in at least priority groupings, the ROC weighting translates these given priorities into appropriate weights. The Commander's time and supporting staff time are precious during operations and the ROC weighting allows weighting with no substantial burden to the Commander and his staff.

Several recent studies and papers have shown this method to be well represented. The method considers the successors in its calculations, but not its predecessors.

$$
w_{i}=\frac{1}{P} *\left(\sum_{j=i}^{N} \frac{1}{p_{j}}\right), j=1, \ldots, N
$$

$i=$ the index of the element

$P=$ the maximum rank value

$p_{j}=$ the rank value of the $\mathrm{j}^{\text {th }}$ element

$N=$ the total number of elements being considered

$w_{i}=$ the relative value of the $\mathrm{i}^{\text {th }}$ element 
Rank Order Centroid methods are very accurate for capturing both partial rank ordering information as well as attribute weights (Barron and Barrett 1996). While ratio weights using pair-wise comparisons between all missions may yield slightly better results, effort and acceptance of these comparisons are overly burdensome (Jia, Fischer, Dyer 1998).

In a recent study of unmanned aerial vehicles (Ahner 2007) that considered mission effectiveness, ranks were assigned in a two dimensional ranking where ranks were assigned by differing hierarchical unit echelons and a rank within that echelon. The rank order centroid method was compared to seven other weighting methods including a geometric and several linear variations. The rank order centroid method was found to possess the characteristics desired. Linear methods appeared to overly decrease all lower echelon coverage by overemphasizing higher echelon coverage while the geometric variation appeared to overly emphasize higher and lower echelons at the expense of the middle echelons.

Rank Order Centroid weights are assigned to each Named Area of Interest (NAI) and Targeted Area of Interest (TAI) identified in the IPB process. These weights are next used for measuring uncertainty in the situation through an entropy measure.

\subsection{Measuring Uncertainty using Weighted Normalized Entropy}

Information for decision making can be valued in two ways. First, the decisionmaker may not be able to cognitively process all information into their perception. If so, it is useful to provide the information which informs the decision most. In this case, informing the decision is decreasing the uncertainty of the outcome space $(\Omega)$ for a given action $(a \in A)$. Second, the decisionmaker can identify those areas in which they have the most uncertainty and can assign information agents to obtain information that most effectively decreases that uncertainty. In either case, competing information requirements can be valued by the assignment or sensor actions, $a_{I}$, that maximize

$$
H_{a_{I}}=\sum_{a_{I} \in A_{I}}\left(\sum_{j \in P I R} w_{j} \frac{\left(-\sum_{i} p_{i j} \log p_{i j}\right)}{\max e n t_{j}}\right)
$$

where we sum over the set of all actions in the information gaining action space, $a_{I} \in A_{I}$; sum over the set of all Priority Intelligence Requirements, PIR, using the rank order centroid method to determine the weights, $w_{j}$; and use an entropy measure $-\sum_{i} p_{i j} \log p_{i j}$, to measure the decrease in uncertainty which is normalized by the unweighted maximum entropy for that PIR, max ent ${ }_{j}$, which is typically given when each possible outcome for a single PIR is considered equally likely (no certainty to any outcome). We refer to $H_{a_{I}}$ as the a priori action entropy, or in other words, the expected resulting entropy of assigning ISR assets to a particular mission measured before the ISR outcomes of the 'experiment' are known.

Entropy measures have various advantages. Among them are that entropy is a continuous functions so that as information is received there are no sudden jumps in the entropy measure, order that the information is received does not alter the change in entropy, entropy as a maximum value where all outcomes are considered equally likely, and the measure is additive. The main disadvantage of entropy is that it is defined in terms of the mathematics of probability distributions and cannot be considered a measure of the importance of information.

The weighting from the ROC method accounts for the importance of information to each PIR while the Bayesian update of the probability accounts for the capabilities of the sensors. For each available Intelligence, Surveillance, and Reconnaissance (ISR) asset the amount that it reduces uncertainty can be determined using the understanding of the capabilities of the ISR asset, the mission to be observed, and the environmental conditions. Sensor placement must be considered when determining the amount that it reduces uncertainty. 


\subsection{ISR Assignment}

ISR assignment has the goal of reducing entropy as weighted by the Commander's priorities. Interpreting this weighted entropy as a measure of uncertainty, information can be quantified as the difference of the entropy between two given probability distributions of a random process. The information gain, $I$, achieved on a target for an efficient assignment of ISR assets is then $I=H_{\varnothing}-\min _{a_{I} \in A} H_{a_{I}}$ where $H_{\varnothing}$ is the entropy if no ISR assignment occurred, also the actual current entrory measure of the system, and $\min _{a_{I} \in A} H_{a_{I}}$ is the minimum entropy as determined by an assignment algorithm. Following some passage of time, ISR assets may once again become available while others may still be functionally assigned to requirements. An ISR assignment can again take place under the constraint of already assigned ISR assets being unavailable and available ISR assets requiring new assignment. The dynamic nature of this assignment through time and across different phases of the operation can be difficult. For now, we use a myopic approach to assigning sensors and leave the dynamic programming of ISR assets for another time.

\subsection{ISR Outcomes and the Next Iteration}

Outcomes from completed ISR assignments can take on many values or states. For this paper, we restrict these outcomes to detection or no detection. If no detection occurs, then uncertainty is decreased and the entropy measure is adjusted accordingly. If a detection occurs, since we allow no false detects, a PIR may be satisfied. If a PIR is satisfied then no more sensor assets will be assigned to cover that PIR since its entropy is zero.

For each outcome, the weighted entropy measures of the system may change. In contrast to the a priori entropy discussed earlier, this is post outcome entropy or actual entropy of the system. This actual entropy, along with parameters from the current state of the system, is used as the base entropy for the next iteration.

\section{EXPERIMENTATION \& RESULTS}

Consider a scenario with four priority intelligence requirements (PIR) and five sensors. We do not present the detailed scenario for brevity. NAIs are associated with each PIR indicating the identified sources of where that information may be obtained. The PIR with potential states are shown in Table 1 along with their ranked priority. The commander has determined that the missile launcher is their top priority, followed by location of the enemy brigade tactical operations center (TOC), presence of possible reinforcements via identified enemy avenues of approach, and, finally, the location of the enemy forces.

Table 1: Scenario Priority Intelligence Requirements, Weights, and Maximum Entropy

\begin{tabular}{|c|l|c|c|c|}
\hline PIR & \multicolumn{1}{|c|}{ Possible States } & Priority & Max Entopy & ROC Weight \\
\hline $\begin{array}{c}\text { PIR \# 1 Location } \\
\text { of Enemy Forces }\end{array}$ & $\begin{array}{l}\text { Presence within NAI 1 } \\
\text { Presence within NAI 2 } \\
\text { Presence within NAI 3 } \\
\text { No enemy present }\end{array}$ & 4 & 0.6021 & 0.1200 \\
\hline $\begin{array}{c}\text { PIR \#2 } \\
\text { Reinforcements } \\
\text { Enter AO }\end{array}$ & $\begin{array}{l}\text { Reinforcements thru NAI 4 } \\
\text { Reinforcements thru NAI 5 } \\
\text { No reinforcements }\end{array}$ & 3 & 0.4771 & 0.2800 \\
\hline $\begin{array}{c}\text { PIR \#3 Location of } \\
\text { Exocet Missile } \\
\text { Launcher }\end{array}$ & $\begin{array}{l}\text { Presence within NAI 1 } \\
\text { Presence within NAI 2 } \\
\text { Presence within NAI 3 } \\
\text { No launcher present }\end{array}$ & 1 & 0.6021 & 1.0000 \\
\hline $\begin{array}{c}\text { PIR \#4 Determine } \\
\text { Enemy BDE TOC } \\
\text { location }\end{array}$ & $\begin{array}{l}\text { Presence within NAI 1 } \\
\text { Presence within NAI 2 } \\
\text { Presence within NAI 3 } \\
\text { No enemy present }\end{array}$ & 2 & 0.6021 & 0.5200 \\
\hline
\end{tabular}


Using $-\sum_{i} p_{i j} \log p_{i j}$ to calculate Max entropy when each possible state is equally likely and (1) to determine the ROC weights yields the final columns of Table 1 .

Sensors have differing capabilities dependent on target type and location. For each Sensor, NAI, and target activity, the associated probability of detection is given in Table 2 . For instance, a moving target can easily be detected by a synthetic aperature radar moving target indicator sensor where as a stationary target is much more difficult to detect by that sensor. Likewise, a target emitting a signal will be more likely to be detected by a electronic intelligence sensor and so on. False detections and other confounding detection states will not be considered but can be addressed by the methodology in this paper.

Table 2: Sensor Detection Capabilities for each Named Area of Interest (NAI)

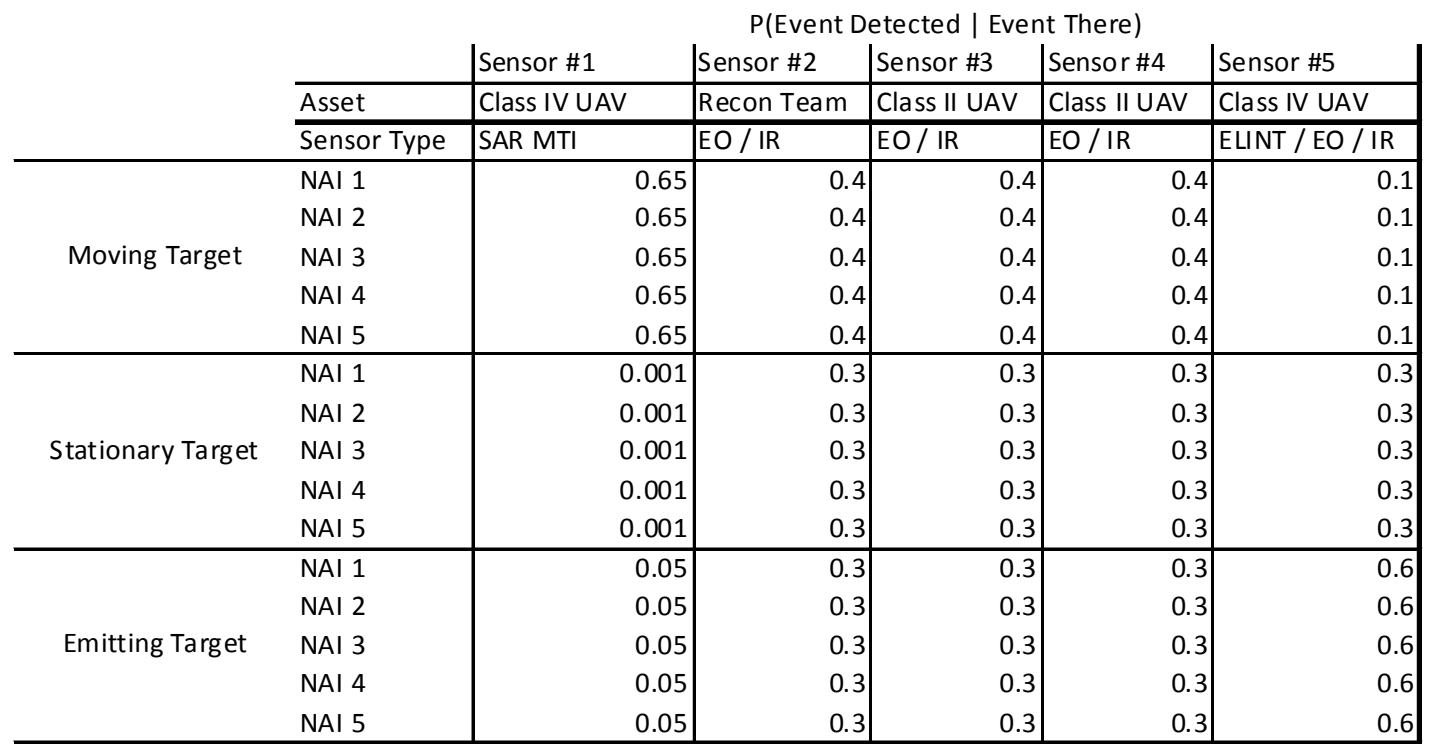

Applying (2) using the parameter values from Table 1 and Table 2 allows us to determine the decrease of the weighted entropy caused by the assignment of each sensor to a NAI. Table 3 shows the decrease in weighted entropy by assigning a sensor to a given NAI. In this case, we assign sensor \#5 to NAI 1.

Table 3: Decrease in Weighted Entropy by Sensor Assignment 1

\begin{tabular}{|l|c|c|c|c|c|}
\cline { 2 - 6 } \multicolumn{1}{c|}{} & Sensor \#1 & Sensor \#2 & Sensor \#3 & Sensor \#4 & Sensor \#5 \\
\hline NAI 1 & 0.0000 & 0.0270 & 0.0270 & 0.0270 & 0.0546 \\
\hline NAI 2 & 0.0000 & 0.0270 & 0.0270 & 0.0270 & 0.0546 \\
\hline NAI 3 & 0.0000 & 0.0270 & 0.0270 & 0.0270 & 0.0546 \\
\hline NAI 4 & 0.0390 & 0.0140 & 0.0140 & 0.0140 & 0.0009 \\
\hline NAI 5 & 0.0390 & 0.0140 & 0.0140 & 0.0140 & 0.0009 \\
\hline
\end{tabular}

Since entropy values have changed, we consider the new entropy values for the next assignment. Weighted entropy values are once again calculated as given in Table 4 and the process continues until all sensors are assigned. No detections occur during this process since no sensor outcomes have yet occurred. Once outcomes do occur, the available sensor is once again assigned according to its maximum decrease in weighted entropy. 
Table 4: Decrease in Weighted Entropy by Sensor Assignment 2

\begin{tabular}{|l|c|c|c|c|c|}
\cline { 2 - 6 } \multicolumn{1}{c|}{} & \multicolumn{5}{c|}{ Decrease in Weighted Entropy } \\
\cline { 2 - 6 } \multicolumn{1}{c|}{} & Sensor \#1 & Sensor \#2 & Sensor \#3 & Sensor \#4 & Sensor \#5 \\
\hline NAI 1 & 0.0001 & 0.0545 & 0.0545 & 0.0545 & \\
\hline NAI 2 & 0.0001 & 0.0530 & 0.0530 & 0.0530 & \\
\hline NAI 3 & 0.0001 & 0.0530 & 0.0530 & 0.0530 & \\
\hline NAI 4 & 0.0390 & 0.0140 & 0.0140 & 0.0140 & \\
\hline NAI 5 & 0.0390 & 0.0140 & 0.0140 & 0.0140 & \\
\hline
\end{tabular}

In comparing Table 3 and 4, it first appears that weighted entropy has increased instead of decreased. This not the case. Since no outcome has yet been determined, the weighted entropy associated with no enemy activity, which is not shown since no sensor can be assigned to it, has decreased. This results in a weighted entropy changing from .12 to .1180 for PIR \#1, no change for PIR \#2, from 1 to .9835 for PIR \#3, and from .52 to .4839 for PIR \#4 due to the assignment of Sensor \#5 to NAI 1 . The sum of these weighted entropy measures is 0.0546 , the value of assigning Sensor \#5 to NAI 1 in Table 3 . Each assignment of a sensor only decreases the total weighted entropy for the case where the outcome is a detection or no detection and no false detections are allowed.

\section{CONCLUSIONS}

The methodology of assigning sensor assets presented here offers a robust means to determine the tradeoff of sensors being assigned to missions within simulations. The methodology uses a framework constructed from a cognitive model and Army doctrine. Within this framework elements of decision theory, ranking of priorities, information theory, and a new weighted entropy measure are used to produce reasonable sensor assignments within combat models. While in real life this assignment of sensors requires the skillful art of an intelligence analyst, the process presented in this paper produces reasonable sensor assignments to which the intelligence analyst would make only minor modifications.

The experiment and results section illustrates the methodology using a normalized weighted entropy measure to produce assignments that rely on the mission priorities, current uncertainty of Priority Intelligence Requirements (PIR), and availability and capabilities of the sensors.

\section{ACKNOWLEDGMENTS}

The author gratefully acknowledges support received from the Army Research Office as part of the Sensor to Commander Metrics project.

\section{REFERENCES}

Ahner, D., A. Buss, and J. Ruck. 2006. Assignment Scheduling Capability for Unmanned Aerial Vehicles, In Proceedings of the 2006 Winter Simulation Conference, eds. L. R. Perrone, F. P. Wieland, J. Liu, B. G. Lawson, D. M. Nicol, and R. M. Fujimoto, 1349-1356. Piscataway, New Jersey: Institute of Electrical and Electronics Engineers, Inc.

Ahner, D. 2007. U.S. Department of the Army, TRADOC Analysis Center, Assignment Capability for UAS Methodology, Monterey, CA: Technical Report TRAC-M-TD-07-011.

Ahner, D., J. Alt, F. Baez, J. Jackson, S. M. Sanchez, and T. Seitz. 2008. Incorporating Information Networks Into Military Simulations, In Proceedings of the 2008 Winter Simulation Conference, eds. S. J. Mason, R. R. Hill, L. Mönch, O. Rose, T. Jefferson, J. W. Fowler, 133-144. Piscataway, New Jersey: Institute of Electrical and Electronics Engineers, Inc.

Army Field Manual 2-0. Intelligence. 2004.

Army Field Manual 6-0. Mission Command: Command and Control of Army Forces. 2003.

Barr, D. and T. Sherrill. 1996. Exploring a Relationship Between Tactical Intelligence and Battle Results, Military Operations Research, 2:3, 1996.

Barron, F.H. and B.E. Barrett. 1996. Decision Quality Using Ranked Attribute Weights. Management Science, 42(11), November 1996, 1515-1523.

Clancey, W.J. 1997. Situated Cognition: on human knowledge and computer representations, Cambridge University Press.

Crain, W.F. 2003. Multi-attribute Weight Determination: Elicitation \& Approximation. Dissertation, George Mason University, Spring 2003. 
Endsley, M., B. Bolte, and D. G. Jones. 2003. Designing for Situation Awareness: An Approach to User-Centered design, CRC Press.

Edwards, W. and F. H. Barron. 1994. SMARTS and SMARTER: Improved Simple Methods for Multiattribute Utility Measurement. Organizational Behavior and Human Decision Processes, 60, 1-20.

Gonzales, D., L. R. Moore, C. G. Pernin, D. M. Matonick, and P. Dreyer. 2001. Assessing the Value of Information Superiority for Ground Forces-Proof of Concept, RAND Report.

Hammitt, J., J. Cave, M. G. Mustafa, and R. B. Valdez. 1991. Research Planning for Food Safety, RAND Report.

Hauser, J.R. and D. Clausing. 1988. The House of Quality, Harvard Business Review, May-June 1988, 63-73.

Howard, R. and J. Matheson. 2005. Influence Diagrams, Decision Analysis, 2(3).

Jia, J., Fischer, G.W., and Dyer, J.S. 1997. Attribute Weighting Methods and Decision Quality in the Presence of Response Error: A Simulation Study, Working Paper.

<http://wWw.mccombs.utexas.edu/faculty/jim.dyer/DA_WP/WP970003.html> (later appeared in Journal of Behavioral Decision Making)

Kahan, J., D. R. Worley, and C. Stasz. 1989. Understanding Commander's Information Needs, RAND Report.

Saaty, T.L. 1980. The Analytic Hierarchy Process: Planning, Priority Setting, Resource Allocation. New York: McGrawHill.

Saaty, T.L. 1982. Decision Making for Leaders. Belmont, CA: Lifetime Learning Publications.

Shattuck, L. and N. Miller. 2006. Naturalistic Decision Making in Complex Systems: A Dynamic Model of Situated Cognition Combining Technological and Human Agents. Organizational Behavior: Special Issue on Naturalistic Decision Making in Organizations. Vol. 27.

Washburn, A. 2001. Bits, Bangs, or Bucks? The Coming Information Crisis, PHALANX, Vol. 34.

Spinuzzi, M. A. 2007. CCIR for Complex and Uncertain Environments, Monograph, School of Advanced Studies, Army Command and General Staff College, Fort Leavenworth, KS.

\section{AUTHOR BIOGRAPHY}

DARRYL AHNER is an Assistant Professor in the Department of Mathematical Sciences at the United States Military Academy, West Point. He received his Ph.D. in Systems Engineering from Boston University. He also received Masters of Science degrees in Applied Mathematics and Operations Research \& Statistics from Rensselaer Polytechnic Institute. His research interests include approximate dynamic programming, discrete event simulation, and sensor information valuation. His email is <darryl.ahnereus.army.mil> 